\title{
Transcatheter closure of atrial septal defect and interatrial communications with a new self expanding nitinol double disc device (Amplatzer septal occluder): multicentre UK experience
}

\author{
K C Chan, M J Godman, K Walsh, N Wilson, A Redington, J L Gibbs
}

Glenfield Hospital, Groby Road, Leicester LE3 9QP, UK K C Chan

Royal Hospital for Sick Children, Edinburgh EH9 1LF, UK

M J Godman

Alder Hey Children's Hospital, Liverpool L12 2AP, UK

K Walsh

Royal Hospital for Sick Children, Glasgow G3 8SL, UK $\mathrm{N}$ Wilson

Brompton Hospital, Sydney Street, London SW3 6NP, UK

A Redington

Yorkshire Heart Institute, Leeds LS1 3EX, UK

J L Gibbs

Correspondence to: Dr Chan.

email: chen.chan@

glenfield-tr.trent.nhs.uk

Accepted for publication 11 January 1999

\begin{abstract}
Objective-To review the safety and efficacy of the Amplatzer septal occluder for transcatheter closure of interatrial communications (atrial septal defects (ASD), fenestrated Fontan (FF), patent foramen ovale (PFO)).
\end{abstract}

Design-Prospective study following a common protocol for patient selection and technique of deployment in all participating centres.

Setting-Multicentre study representing total United Kingdom experience.

Patients-First 100 consecutive patients in whom an Amplatzer septal occluder was used to close a clinically significant ASD or interatrial communication.

Interventions-All procedures performed under general anaesthesia with transoesophageal echocardiographic guidance. Interatrial communications were assessed by transoesophageal echocardiography with reference to size, position in the interatrial septum, proximity to surrounding structures, and adequacy of septal rim. Stretched diameter of the interatrial communications was determined by balloon sizing. Device selection was based on and matched to the stretched diameter of the communication. Main outcome measures-Success defined as deployment of device in a stable position to occlude the interatrial communication without inducing functional abnormality or anatomical obstruction. Occlusion status determined by transoesophageal echocardiography during procedure and by transthoracic echocardiography on follow up. Clinical status and occlusion rates assessed at 24 hours, one month, and three months.

Results-101 procedures were performed in 100 patients (86 ASD, 7 FF, 7 PFO), age 1.7 to 64.3 years (mean (SD), 13.3 (13.9)), weight 9.2 to $100.0 \mathrm{~kg}$ (mean 32.5 (23.5)). Procedure time ranged from 30 to 180 minutes (mean 92.4 (29.0)) and fluoroscopy time from 6.0 to 49.0 minutes (mean $16.1(8.0)$ ). There were seven failures, all occurring in patients with ASD, and one embolisation requiring surgical removal. Immediate total occlusion rate was $20.4 \%$, rising to $84.9 \%$ after 24 hours. Total occlusion rates at the one and three month follow up were $92.5 \%$ and $98.9 \%$, respectively. Complications were: transient ST eleva- tion (1), transient atrioventricular block (1), presumed deep vein thrombosis (1), presumed transient ischaemic attack (1). Conclusions-It appears feasible to close interatrial communications and atrial septal defects up to $26 \mathrm{~mm}$ stretched diameter safely with the Amplatzer septal occluder. Short term results confirm an early high occlusion rate with no major complications. Careful selection of cases based on the echocardiographic morphology of the ASD and accurate assessment of their stretched diameter is of utmost importance. Further experience with the larger devices and longer term results are required before a firm conclusion regarding its use can be made.

(Heart 1999;82:300-306)

Keywords: interatrial communications; atrial septal defect; Amplatzer septal occluder; congenital heart defects

Atrial septal defect (ASD) is a common congenital heart defect with an estimated prevalence of 3.78/10 000 live births ${ }^{1}$ and ranks as the fourth most common form of congenital heart disease $(5.9 \%$ of cases of congenital heart disease in the paediatric population $^{2}$ ). It is, however, one of the most common congenital heart defects in adulthood. ${ }^{3}$ Recent reports on its natural history and surgical treatment have challenged the traditional concepts of this defect and have rekindled interest in its management. ${ }^{4-7}$ In spite of recent controversies regarding surgical treatment, 623 surgical closures of ASD were performed in Great Britain and Ireland in 1995-96. ${ }^{8}$

Following the initial successful report of non-surgical transcatheter closure of ASD, ${ }^{9}$ various devices for transcatheter closure of ASD were introduced and are currently being evaluated. ${ }^{10-14}$ Although the short and medium term follow up of several of these devices has been encouraging, there have been concerns about fatigue fractures of metal struts, ${ }^{15}$ and displacement of the device or perforation of the atrial wall necessitating surgical removal. ${ }^{16}{ }^{17}$ These problems may reflect the design of the devices, which are oversized square shaped occlusion discs or wings to effect occlusion, and also the retrievability of the device during implantation. Furthermore, the delivery systems are often large ( $9 \mathrm{~F}$ to $14 \mathrm{~F}$ long sheath) 


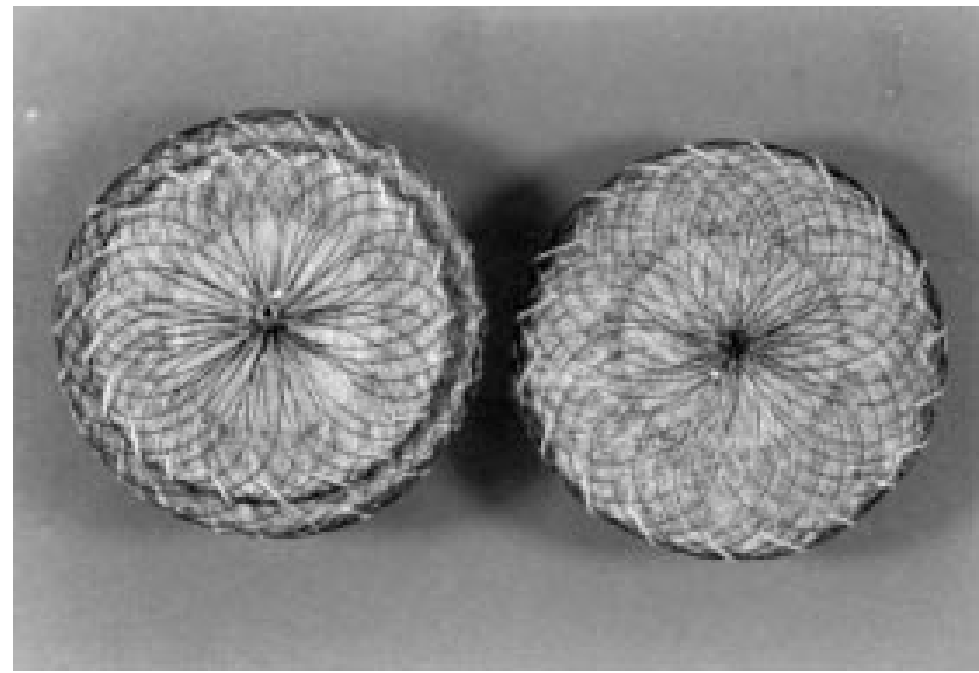

Figure 1 Right atrial and left atrial aspect of the Amplatzer septal occluder.

and the delivery techniques complicated. Recently, a new device-a self expanding circular double disc with a conjoint waist made up of nitinol windings (Amplatzer septal occluder, ASO; AGA Medical Corporation, Golden Valley, Minnesota, USA)-has been introduced for the purpose of non-surgical transcatheter closure of ASD and other interatrial communications. Initial animal studies ${ }^{18}$ and early human experience ${ }^{19}$ have been promising. We report our initial experience and short term outcome with the ASO device for non-surgical transcatheter closure of ASD, patent foramen ovale with presumed paradoxical emboli, and patients with fenestrated Fontan procedure, with reference to its safety and efficacy.

\section{Methods}

All the participating centres followed a common protocol designed by Amplatz. ${ }^{20}$ Approval from respective local ethics committee was obtained in each participating centre.

\section{PATIENT SELECTION}

All patients with a clinically significant fossa ovalis ASD and in whom cardiac surgery was indicated were invited to participate in the trial. Initial selection was based on transthoracic echocardiographic assessment. ASD with a transthoracic echocardiographic diameter of $20 \mathrm{~mm}$ or less with a suitable septal rim of at least $5 \mathrm{~mm}$ from the right pulmonary veins, coronary sinus, superior caval vein, inferior caval vein, and mitral valve were considered suitable. Latterly, with the availability of the larger devices from May 1997, defects with a diameter of up to $26 \mathrm{~mm}$ and a septal rim of at least $7 \mathrm{~mm}$ were considered potentially suitable.

Patients with patent foramen ovale and a history of stroke or transient ischaemic attack with a positive contrast echocardiographic study and patients with fenestrated Fontan procedure were also included in the trial. Informed consent was obtained from all patients or their parents or legal guardian.
DEVICE

A prototype of the device has been described previously. ${ }^{18}$ The ASO device used in the trial is a circular double disc frame with a conjoint waist made of windings of nitinol, a nickeltitanium alloy (fig 1). Nitinol has the properties of super-memory and super-elasticity which make it suitable for the design of the device. The principle of the device is based on the conjoint waist "stenting" the ASD, providing both fixation and occlusion. Polyester inserts are placed within the device to facilitate thrombosis and occlusion. The quoted size of the device is the diameter of the conjoint waist. The diameter of the left atrial disc is $10 \mathrm{~mm}$ larger than the conjoint waist in devices up to $10 \mathrm{~mm}$ (that is, $5 \mathrm{~mm}$ rim) and $14 \mathrm{~mm}$ larger than the conjoint waist in devices greater than $10 \mathrm{~mm}$ (that is, $7 \mathrm{~mm}$ rim). The right atrial disc is $8 \mathrm{~mm}$ greater than the waist in all the devices. The ASO is available in sizes with $1 \mathrm{~mm}$ increments from $4 \mathrm{~mm}$ to $20 \mathrm{~mm}$, and then in $2 \mathrm{~mm}$ increments until the current largest device of $26 \mathrm{~mm}$. Devices up to $10 \mathrm{~mm}$ are made up of nitinol windings of 0.004 inches $(0.10 \mathrm{~mm})$; thicker windings of 0.005 inches $(0.13 \mathrm{~mm})$ are used in devices from $10 \mathrm{~mm}$ to $20 \mathrm{~mm}$, while devices with a diameter greater than $20 \mathrm{~mm}$ have thicker windings of 0.006 inches $(0.15$ $\mathrm{mm}$ ) for added strength. The device is attached to the delivery wire by a central screw thread made of surgical grade stainless steel. This screw attachment is laser welded to the nitinol frame. The device is delivered through a $7 \mathrm{~F}$ or $8 \mathrm{~F}$ kink resistant Teflon long delivery sheath.

\section{TECHNIQUE}

All the procedures were performed under general anaesthesia. Detailed assessment of the size and morphology of the ASD was performed by transoesophageal echocardiography (TOE) with either a biplane or a multiplane probe.

The maximum diameter of the defect, the septal length, and the distance of the edge of the defect from the right pulmonary veins, the orifice of the coronary sinus, and the mitral valve were measured using the transverse plane. The distances from the superior and inferior caval vein were measured from the sagittal or vertical plane. Images of the anterior rim adjacent to the posterior border of the aorta were obtained using both the horizontal and vertical plane.

After a complete saturation and haemodynamic study had been carried out, pulmonary arteriography was performed in the anteroposterior view to exclude anomalous pulmonary venous drainage. This was followed by a right upper pulmonary vein angiography in the four chamber view to visualise the atrial septum. The ASD was sized with a sizing balloon catheter and a precalibrated sizing template. The stretched diameter of the ASD was defined as the diameter of a balloon that can be withdrawn across the ASD with mild resistance and slight deformity. In cases with patent foramen ovale, the defect was also assessed by forward sizing with the sizing balloon advanced 
from the right atrium into the left atrium over a guide wire.

Both fluoroscopy and TOE guidance were used throughout the sizing procedure to ensure proper positioning of the sizing balloon catheter. Device selection was based on and matched to the stretched diameter of the ASD. Thus the selected ASO device was either the same size as the stretched diameter or $1 \mathrm{~mm}$ larger. The selected ASO device was attached to the delivery wire by the screw mechanism and was loaded by withdrawing into the loader by traction on the delivery wire. The collapsed ASO device was then advanced through the long sheath that had previously been positioned in the left atrium. Under fluoroscopic control the left atrial disc was extruded by advancing the delivery wire. The sheath and the delivery wire were withdrawn in unison until resistance was met when the extruded left atrial disc was apposed to the atrial septum. The ASO device was then fully deployed by withdrawing the sheath over the delivery wire to extrude the right atrial disc.

Once the device was fully deployed across the ASD, its position and stability were assessed by fluoroscopy and TOE. Its position was deemed optimal if the device was stable and did not obstruct the right pulmonary veins, coronary sinus, caval veins, or the mitral valve. Any residual shunt was documented using colour flow Doppler on TOE. The ASO device was then released by anticlockwise rotation of the delivery wire. Before this release, the device could easily be retrieved by withdrawing it into the sheath by traction on the delivery wire. A final assessment of the position of the device was performed by TOE following its release. A repeat haemodynamic study and pulmonary angiography in a four chamber view were performed (fig 2).

The patients were returned to the general ward and discharged the next day on an antiplatelet dose of aspirin. A repeat transthoracic echocardiography, electrocardiography, and chest $x$ ray were performed the next day before discharge. Follow up was arranged for one month, three months, and one year after the implant.

ROLE OF TRANSOESOPHAGEAL

ECHOCARDIOGRAPHY

TOE is essential and allows complete and accurate assessment of the morphology of the ASD without interfering with the sterility of the operative field or obstructing fluoroscopy. The septal rims are well delineated and the distance from the edge of the defect to the right pulmonary veins, superior and inferior caval veins, coronary sinus, and mitral valve were easily measured (figs 3 and 4). Variations of the atrial septum such as fenestrations and aneurysm may not be seen by transthoracic echocardiography, particularly in adult patients, but are well identified by TOE. Fenestrations in the atrial septum complicate the procedure if sizing is inadvertently performed through the smaller defect. Thus in the presence of fenestrations it is imperative that the guide wire, sizing balloon, and the delivery sheath are shown to be
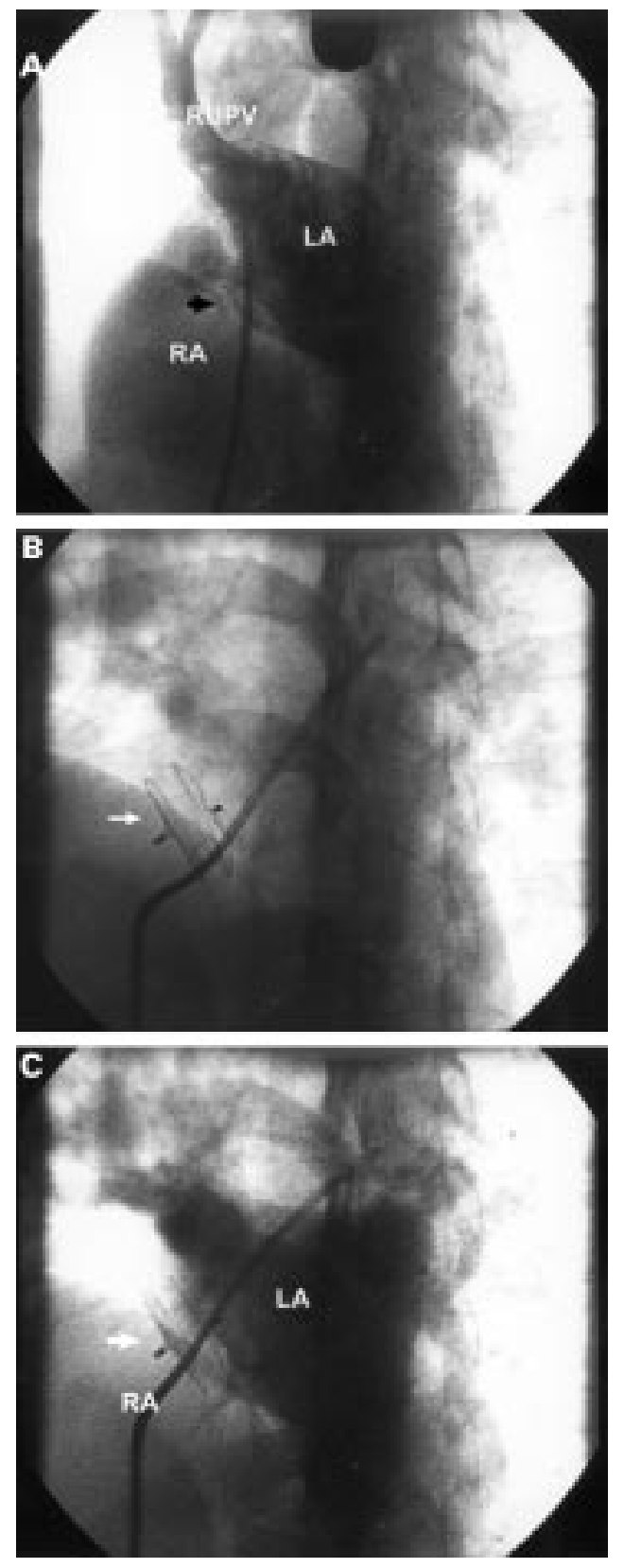

Figure 2 (A) Right upper pulmonary vein angiogram in four chamber view showing atrial septal defect with "septal aneurysm.” (B) Four chamber view showing Amplatzer septal occluder in place. (C) Venous phase following pulmonary angiography in four chamber view after device deployment showing no residual leak. $L A$, left atrium; $R A$, right atrium; RUPV, right upper pulmonary vein. Black arrow, atrial septal defect; white arrow, septal occluder.

through the major defect. Following deployment of the device, TOE was used to assess device position, its relation to the surrounding structures, and its stability (figs 3 and 4). Residual shunts are also best demonstrated by TOE.

DATA COLLECTION AND ANALYSIS

All data were collected on a set data sheet, collated by a single coordinator, and entered into a common database for analysis. Descriptive statistical data are presented as mean (SD).

OUTCOME MEASURES

Safety and efficacy is defined as follows: 

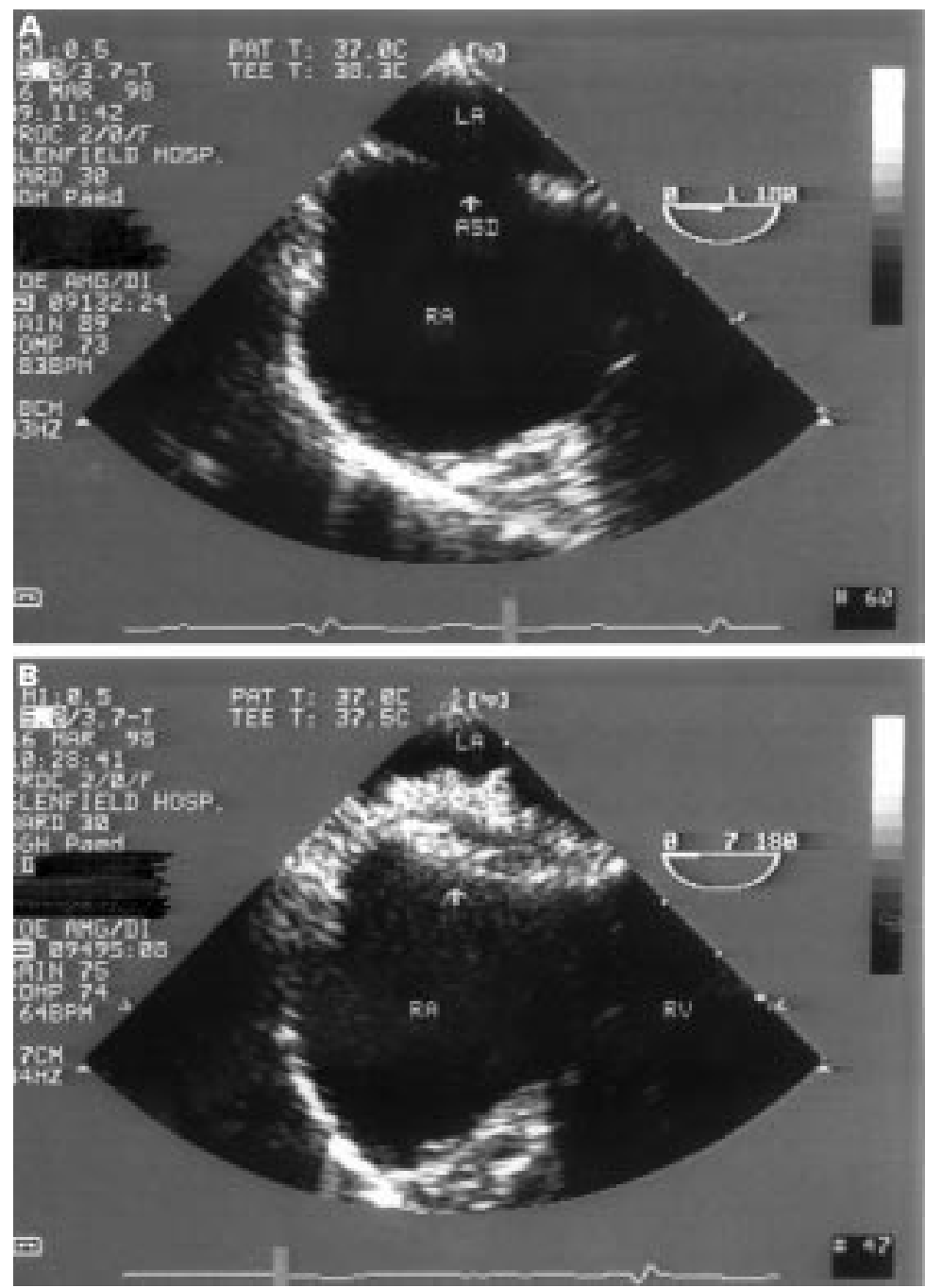

Figure 3 Transoesophageal echocardiogram in transverse plane showing atrial septal defect before occlusion (A) and after occlusion by the Amplatzer septal occluder (B). ASD, atrial septal defect; $L A$, left atrium; $R A$, right atrium; $S V C$, superior vena cava. White arrow, septal occluder. seven with a patent foramen ovale, and a further seven with a fenestrated Fontan procedure. They ranged in age from 1.7 to 64.3 years (mean (SD) $13.2(13.9)$ ) and in weight from 9.2 to $100.0 \mathrm{~kg}(32.5$ (23.5)). Procedural time ranged from 30.0 to 180.0 minutes (mean (SD) 92.4 (29.0)) with screening times from 6.0 to 49.0 minutes $(16.1(8.0))$. There were 93 successful implants and seven failures. The frequency distribution of the devices used are listed in fig 5 . The smaller devices were used in patients with a fenestrated Fontan procedure.

\section{FAILURE TO IMPLANT}

There were seven failures to implant, all occurring in patients with ASD. Two failures occurred early in the trial. The stretched diameters of the ASD in these two patients were assessed to be $22 \mathrm{~mm}$ and $19.5 \mathrm{~mm}$ and the largest devices available then, with a diameter of $20 \mathrm{~mm}$, were deployed. Although the devices were deployed correctly, they could easily be dislodged while still attached to the delivery wire. They were considered unstable and were withdrawn into the delivery sheath and retrieved.

Two larger devices (22 $\mathrm{mm}$ and $24 \mathrm{~mm}$ ) were also not released and were withdrawn when they were seen on TOE to obstruct the superior caval vein and the mitral valve, respectively.

The position of two other devices was considered unsuitable or unstable after implantation. These two devices appeared to have tilted downwards from tension of the delivery wire, resulting in the superior part of the left atrial disc prolapsing through the defect into the right atrium. One of these patients had a thin floppy atrial septal aneurysm in addition to the ASD and this may have been a contributing factor. Again both these devices were withdrawn while still attached to the delivery wire.

In the last patient in whom we failed to implant, the right atrial disc did not configure correctly when deployed and assumed a twisted shape. This device was also withdrawn before release.

\section{COMPLICATIONS}

There was one complication of embolisation occurring in a patient with a $26 \mathrm{~mm}$ device that appeared to be stable at the time of implantation but embolised to the main pulmonary artery overnight. The device was retrieved at surgery and the ASD closed at the same time the following day without any further complication.

There were four other documented complications. One patient developed transient ST segment elevation in the inferior leads during deployment of the device. This was attributed to a small air embolus. It resolved spontaneously and was not associated with any haemodynamic derangement or permanent ECG changes. Another patient developed transient atrioventricular block with an acceptable ventricular escape rate during manipulation of the catheter in the right ventricle. Again this did not result in any haemodynamic compromise. 

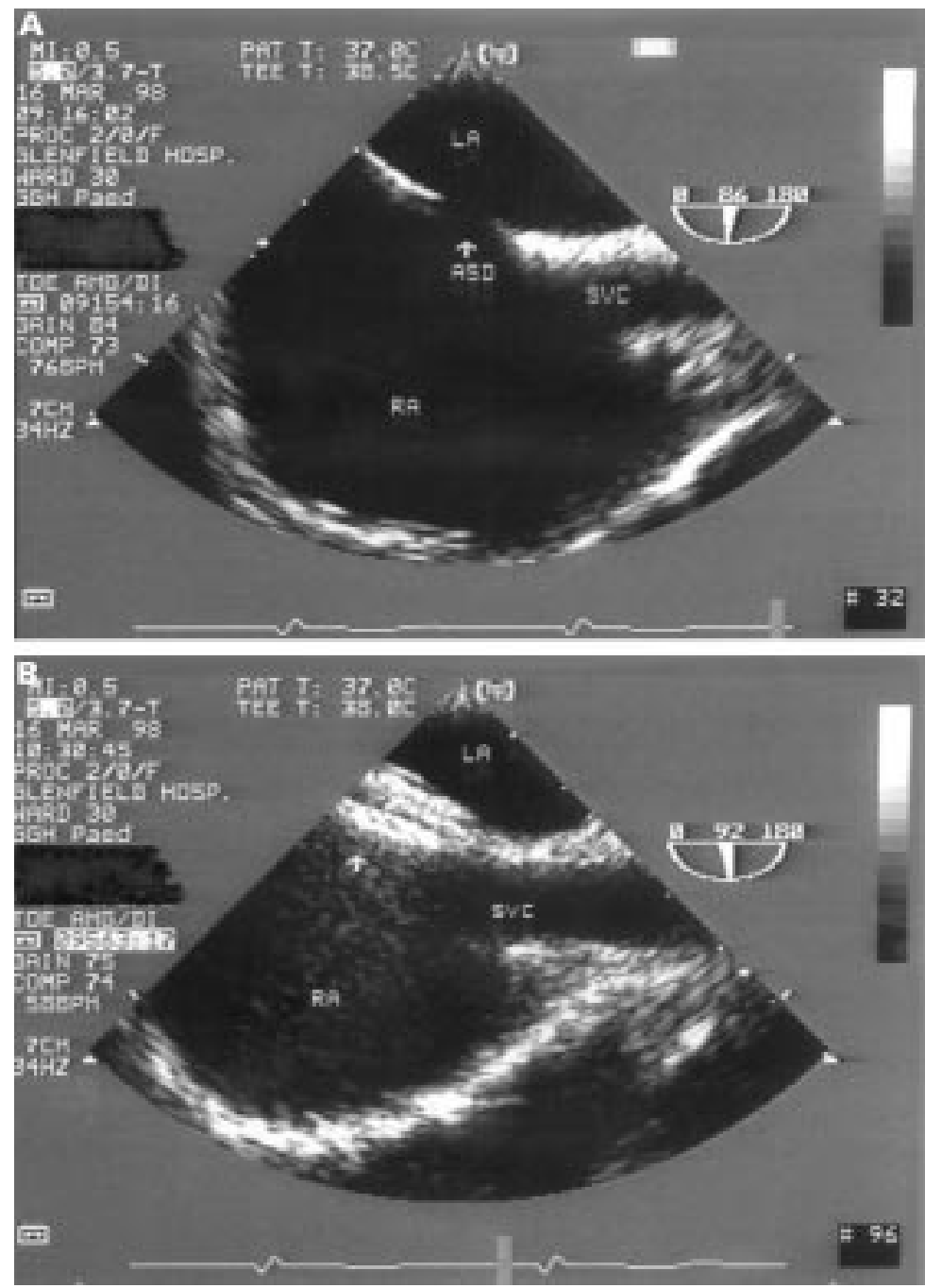

Figure 4 Transoesophageal echocardiogram in sagittal view showing atrial septal defect in relation to superior vena cava before occlusion $(A)$ and after occlusion by the Amplatzer septal occluder (B). ASD, atrial septal defect; $L A$, left atrium; $R A$, right atrium; $S V C$, superior vena cava. White arrow, septal occluder.

An adult patient developed signs and symptoms of deep vein thrombosis in the contralateral leg one week after the procedure. This resolved following treatment with heparin and anticoagulation. The most worrying complication occurred in an adult patient with a $24 \mathrm{~mm}$ device. He complained of transient loss of vision over the lateral aspect of the left eye 10 days after the procedure. There was no neurological deficit when he was admitted for observation. Repeat TOE on admission showed the

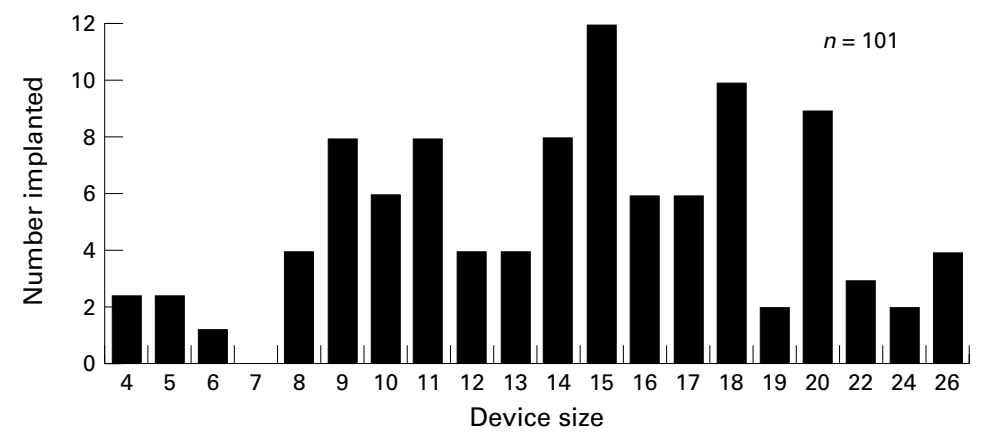

Figure 5 Frequency distribution of devices implanted by size.
ASO device to be in place with no evidence of thrombus. This patient was started on warfarin and recovered completely with no further recurrences of his symptoms.

There was no mortality nor were any patients referred for emergency surgery owing to malposition of the device.

IMMEDIATE AND SHORT TERM OUTCOME

Of all 93 patients in whom the ASO was successfully implanted, 19 (20.4\%) achieved total occlusion at implantation, as assessed by TOE. In the majority of the patients, a small diffuse residual leak through the central portion of the device could be seen. However, by 24 hours after implantation, the total occlusion rate had risen to $84.9 \%(79 / 93)$ as evidenced by transthoracic echocardiography before discharge. Echocardiography at the one month follow up showed a total occlusion rate of $92.5 \%$ (86/93) with only trivial residual shunt in the others. The total occlusion rate had risen to $98.9 \%(92 / 93)$ by three months, with one result pending. In the patients with patent foramen ovale or fenestrated Fontan procedure, complete occlusion were achieved by the one month follow up. If we exclude these patients, the occlusion rates in the patients with ASD were $14.0 \%(11 / 79), 84.8 \%$ (67/79), $92.4 \%(73 / 79)$, and $98.7 \%(78 / 79)$ at implantation, 24 hours, one month, and three months, respectively.

All the patients remained well with no complications or new symptoms at their one or three month follow up.

\section{Discussion}

Most devices designed for ASD closure depend on two oversized discs which appose each other to effect closure. Apart from the Das device, ${ }^{14}$ none is truly self centring. In spite of encouraging results with some of the devices, their routine use has not become widespread. Concerns have been expressed over large delivery systems, complicated technique, significant complications including risk of air embolism, late perforation, unacceptable residual leak, and device failure (unbuttoning and strut fractures). One important concern with most devices is the lack of retrievability during deployment, resulting in the need for surgical retrieval should there be malpositioning of the device.

The ASO employs the concept of closing the ASD by stenting the defect with its conjoint waist. The device is therefore truly self centring and achieves fixation and stability by stenting the defect. Extra stability is provided by the two atrial discs. The polyester inserts in the atrial disc and the conjoint waist facilitate closure by promoting thrombosis and subsequent neoendothelium formation. The device was designed to be easily retrievable until it is released from the delivery wire, thereby allowing removal in the event of malpositioning. This was easily demonstrable in the seven cases where the devices were implanted incorrectly or in an unstable position. This retrievability has the advantage of avoiding emergency surgery in 
these problematic cases. The overall technique appears simpler than with the other existing devices and therefore has a shorter learning curve.

Careful patient selection is important. There must be accurate assessment of the morphology of the ASD with respect to its position in the atrial septum and its proximity to surrounding structures such as the superior caval vein, inferior caval vein, coronary sinus, right pulmonary veins, and the atrioventricular valves. Selection of the correct size of device depends on accurate assessment of the stretched diameter of the ASD, as the device is matched to the size of the stretched ASD diameter. This is currently done using a balloon catheter filled with dilute contrast and a precalibrated template. During the sizing process, care must be taken not to undersize oval defects, as resistance from the minor diameter may be felt as the balloon is withdrawn across the defect. This error may be minimised by using TOE to visualise the defect during balloon sizing. Correct positioning and stability of the ASO device immediately after implantation must be assessed using TOE before it is finally released.

One of the aims of device closure of an ASD is to cause abolition of the shunt to a degree comparable with surgical closure. However, the design of the device with its nitinol wire mesh, and heparinisation during the procedure, results in the high incidence of residual leaks immediately after implantation. These residual leaks were easily detected by TOE; they occurred through the central conjoint waist and were either trivial or small enough not to be haemodynamically significant. TOE has a high sensitivity for detecting residual leaks compared with transthoracic scans, which were used for assessment after implantation and on follow up. Thus it must be accepted that the follow up echocardiographic assessments were made within the limitations of the sensitivity of transthoracic echocardiography for detecting trivial leaks. Furthermore the fine wire mesh of the device may cause echocardiographic artefacts that make interpretation difficult. Nevertheless it is encouraging that by three months after implantation, almost all the defects were either totally closed or showed at most a trivial residual shunt. Recovery of the right ventricular volume overload is expected to occur but a longer term follow up is required.

In the early part of the study, only devices up to a diameter of $20 \mathrm{~mm}$ were available. This limited the size of defects that could be closed using the ASO. Since May 1997, devices up to $26 \mathrm{~mm}$ have become available (currently devices up to $34 \mathrm{~mm}$ are available for use in large ASD, but were not available at the time of this study). If the larger devices were available we might have been successful in closing two of the larger ASD in early part of the study. The design of the larger devices remains similar but owing to their size they are delivered through a larger $8 \mathrm{~F}, 9 \mathrm{~F}$, or even $10 \mathrm{~F}$ sheath. It is important to assess the larger devices after implantation by TOE before release because of increased risk that the left atrial disc will impinge upon the surrounding structures. This was seen in two of the patients in our series. It was not surprising that with a larger ASD there was attenuation of the limbus, resulting in a smaller surrounding rim. ${ }^{21}$ The superior aspect of the left atrial disc may prolapse across the defect into the right atrium with a large anteriorly placed ASD. This is caused by a change in the orientation of the device from the downward tension exerted by the delivery wire. If released, these devices will most certainly embolise as fixation is incomplete. The operator must watch out for this potential complication, which is best shown by TOE from the sagittal plane. Non-turbulent colour flow Doppler signal from left atrium to right atrium across the edge of the device is a further clue that the left atrial disc had prolapsed. This was identified on retrospective review of the recorded TOE information in the case that embolised. To minimise this complication, part of the central waist was extruded together with the left atrial disc before engaging the device onto the atrial septum. This manoeuvre allows some self centring before deployment of the right atrial disc. It was noteworthy that anteriorly located defects that abut on to the posterior border of the ascending aorta could still be closed securely with the ASO. The morphological aspects of the fixation of the ASO in these defects must be carefully studied and the effect of the device on the aortic root needs to be clarified in further follow up studies.

The haemodynamic advantage of closing a significant ASD is well recognised. However, whether there is improvement or prevention of clinical deterioration after surgical closure in asymptomatic patients has been debated. ${ }^{45}$ Recent evidence suggests there is improvement in exercise tolerance and aerobic capacity following surgical closure. ${ }^{7}$ Non-surgical closure by interventional cardiac catheter has a potential advantage in that the same haemodynamic benefit may be achieved with no scarring to the chest or atrial myocardium. This is a considerable advantage and tips the balance in favour of closing a significant ASD even if the patient is asymptomatic.

It has been estimated that only about $50 \%$ of ASD in the fossa ovalis may be suitable for transcatheter closure. ${ }^{22}$ This estimate was based on the "Clam-Shell" ASD closure device. However, with further experience and new device development, the proportion of defects suitable for device closure may increase.

Early experience with the ASO suggests that safe closure of an ASD of up to $26 \mathrm{~mm}$ in diameter is feasible if it is located in a suitable position. The ASO device is also suitable for closure of patent foramen ovale and fenestrations after the Fontan procedure. Once deployed the ASO device has a high early occlusion rate. Although the device shows promise, further experience with the larger devices and longer term results are required before a firm conclusion regarding its regular use can be made.

We acknowledge the assistance of Mrs A-M Griffin and Mrs C Norman in collating the data. 
1 Emmanoulides GC, Allen HD, Riemenschneider TA, et al, eds. Heart disease in infants, children and adolescents, including the fetus and young adults. Baltimore: Williams and Wilkins, 1995:60-9.

2 Dickinson DF, Arnold R, Wilkinson JL. Congenital heart disease among 160,480 liveborn children in Liverpoo 1960 to 1969. Implications for surgical treatment. Br Heart f 1981;46:55-62.

3 Perloff JK, Child JS, eds. Congenital heart disease in adults. Philadelphia: WB Saunders, 1991:21-59.

4 Shah D, Azhar M, Oakley CM, et al. Natural history of secundum atrial septal defect in adults after medical or surgical treatment: a historical prospective study. Br Heart $\mathcal{f}$ 1994;71:224-8

5 Ward C. Secundum atrial septal defect: routine surgical treatment is not of proven benefit. Br Heart F 1994;71:219 23.

6 Konstantinides S, Geibel A, Olschewski M, et al. A comparison of surgical and medical therapy for atrial septal comparison of surgical and medical therapy for a

7 Helber U, Baumann R, Sebolt H, et al. Atrial septal defect in adults: cardiopulmonary exercise capacity before and 4 months and 10 years after defect closure. $\mathcal{F} \mathrm{Am}$ Coll Cardio 1997;29:1345-50.

8 UK Cardiac Surgical Register, 1995/96. London: Society of Cardiothoracic Surgeons of Great Britain and Ireland, 1996.

9 King TD, Mills NL. Secundum atrial septal defects: non operative closure during cardiac catheterisation. $\mathcal{F} A M A$ 1976;235:2506-9.

10 Rashkind WJ. Transcatheter treatment of congenital heart disease. Circulation 1983;67:711-16.

11 Lock JE, Rome JJ, Davis R, et al. Transcatheter closure of atrial septal defects: experimental studies. Circulation 1989; 79:1091-9.

12 Sideris EB, Sideris SE, Thanopoulos BD, et al. Transvenous atrial septal defect occlusion by the buttoned device. $A m \mathcal{F}$ Cardiol 1990;66:1524-6.
13 Hausdorf G, Schneider M, Franzbach B, et al. Transcatheter closure of secundum atrial septal defects with the atrial septal defect occlusion system: initial experience in children. Heart 1996;75:83-8

14 Das GS, Voss G, Jarvis G, et al. Experimental atrial septal defect closure with a new, transcatheter, self-centering device. Circulation 1993;88:1754-64.

15 Schlesinger AE, Folz SJ, Beekman RH, et al. Transcatheter atrial septal defect occlusion devices: normal radiographic appearances and complications. F Vasc Intervent Radiol appearances an

16 Rao PS, Sideris EB, Hausdorf G, et al. International experience with secundum atrial septal defect occlusion by the buttoned device. Am Heart 7 1994;128:1022-35.

17 Agarwal SK, Gosh PK, Mital PK. Failure of devices used for closure of atrial septal defects: Mechanisms and management. F Thorac Cardiovasc Surg 1996;112:21-6.

18 Melhem JAS, Gu X, Titus JL, et al. Transvenous closure of secundum atrial septal defects. Preliminary results with a new self-expanding Nitinol prosthesis in a swine model. Circulation 1997;95:2162-8.

19 Bjornstad PG, Masura J, Thaulow E, et al. Interventional closure of atrial septal defects with the Amplatzer ${ }^{\mathrm{tm}}$ device: first clinical experience. Cardiology in the Young 1997; 277-83.

20 Closure of atrial septal defect (ASD) by the Amplatzer ${ }^{\mathrm{TM}}$ septal occluder. Investigational plan. Golden Valley, MN: AGA Medical Corporation, 1996.

21 Rosenquist GC, Sweeney LJ, Ruckman RN, et al. Atrial septal thickness and area in normal heart specimens and in those with ostium secundum atrial septal defects. $\mathcal{F}$ Clin Ultrasound 1979;7:345-8.

22 Chan KC, Godman MJ. Morphological variations of fossa ovalis atrial septal defects (secundum): feasibility for transcutaneous closure with the clam-shell device. Br Heart $\mathcal{F}$ 1993;69:52-5. 